\title{
Toll-Like Receptor Expression of Mesenchymal Stem Cells Derived from Granulocyte Colony-Stimulating Factor Treated Bone Marrow Donors
}

Tekin Aksu', [MD]

ORCID: 0000-0003-4968-109X

Neslihan Karakurt ${ }^{1}$, [MD]

ORCID: 0000-0001-5487-9485

İrem $A k a r^{2},[M D]$

ORCID: 0000-0002-3073-5898

Yasin Köksal ${ }^{3},[\mathrm{MD}]$

ORCID: 0000-0003-0797-9568

Fatih M. Azık', [MD]

ORCID: 0000-0001-5715-4244

Meltem Özgüner ${ }^{3},[\mathrm{MD}]$

ORCID: 0000-0003-0364-5907

Duygu Uçkan Çetinkaya ${ }^{1,2}$, [MD]

ORCID: 0000-0003-3593-6493

'Pediatric Hematology, Ankara Children's Hematology Oncology Education and Research Hospital, Ankara, Turkey.

${ }^{2}$ Hacettepe University Center for Stem Cell Research, Ankara, Turkey; Hacettepe University Graduate School of Health Sciences, Department of Stem Cell Sciences, Ankara, Turkey.

${ }^{3}$ Stem Cell Research Laboratory, Ankara Children's Hematology Oncology Education and Research Hospital, Ankara, Turkey.

Corresponding Author: Tekin Aksu

Pediatric Hematology, Ankara Children's Hematology Oncology Education and Research Hospital, Ankara, Turkey.

E-mail: tekinaksu@gmail.com

\section{w ABSTRACT Con}

Objective: The present study was planned to examine the expression of Toll-like receptors on human marrow-derived mesenchymal stem cells as a result of in-vivo exposure to granulocyte colony-stimulating factor with or without exposure of the cells to Toll-like receptors agonists.

Materials and Methods: Toll-like receptor 2, 3, and 4 expressions of mesenchymal stem cells obtained from healthy human bone marrow donors exposed to in-vivo granulocyte colony-stimulating factor were analyzed, and granulocyte colony-stimulating factor untreated donors served as controls. Also, mesenchymal stem cells were stimulated in-vitro by Toll-like receptor agonists to observe the changes in the expression of the Toll-like receptors.

Results: Mesenchymal stem cells obtained from both granulocyte colony-stimulating factor exposed or unexposed donors showed a low level of Toll-like receptor 2, 4 expressions by flow cytometry, whereas Toll-like receptor 3 expression was higher. Lipopolysaccharide was used as an agonist, but no significant difference was observed in the Toll-like receptor 2, 4 expressions, both in the granulocyte colony-stimulating factor exposed and unexposed groups. Stimulation of cells with Tolllike receptor 3 ligand was associated with a statistically significant decrease in Toll-like receptor 3 expressions, which was more profound in granulocyte colony-stimulating factor unexposed cells.

Conclusion: We have shown that human bone marrow-derived cultureexpanded mesenchymal stem cells express Toll-like receptor 3 , whether in-vivo granulocyte colony-stimulating factor treated or untreated. Besides, the Toll-like receptor 3 agonist's effect in lowering the expression levels was more significant in cells that were not exposed to granulocyte colony-stimulating factor. Additionally, detection of low expression of the pro-inflammatory Toll-like receptor 4 versus higher levels of Tolllike receptor 3 supports literature regarding the immunosuppressive characteristics of marrow-derived mesenchymal stem cells. Modulation of the expression of the Toll-like receptor of mesenchymal stem cells with granulocyte colony-stimulating factor or agonists may have implications in allogeneic mesenchymal stem cell therapies.

Keywords: Mesenchymal stem cell, granulocyte colony-stimulating factor, toll-like receptor, lipopolysaccharide, poly(l:C)

Received: 25 October 2020, Accepted: 4 December 2020

Published online: 31 December 2020 


\section{INRODUCTION}

Mesenchymal stem cells (MSCs) are plastic adherent cells isolated from bone marrow (BM) and other tissues. The International Society for Cellular Therapy suggests fibroblast-like plastic adherent cells isolated from any tissue, termed as multipotent MSCs [1]. MSCs differentiate into osteoblasts, chondrocytes, adipocytes, muscle, ligament, bone marrow stroma, connective tissue, or neural cells in culture conditions or in vivo [2-4]. MSCs could be expanded in vitro and infused to autologous or allogeneic hosts without any significant host immune reactivity. So they are called "immune privileged" cells [3]. MSCs have aroused interest in regenerative medicine and emerged as a promising cellular therapy method for degenerative, chronic inflammatory, autoimmune diseases, and allograft rejection. They home to damaged tissues and contribute to their repair process by secretion of cytokines, chemokines, and extracellular matrix proteins [3, 5-7]. The mechanisms governing stem cell fate, mobilization, and recruitment to engraftment sites are not fully understood.

Toll-like receptors (TLRs) belong to a family of receptors that promote innate immunity activation and appear to regulate adaptive immune responses. TLRs have been associated with Crohn's disease, rheumatoid arthritis, and allograft rejection [7]. So far, ten TLRs have been identified in humans, numbered as TLR1 - 10 [8]. Bacterial or viral molecules as exogenous stimulants and heat shock proteins, extracellular matrix proteins as endogenous stimulants, are recognized by different TLRs [8]. TLR agonist stimulation leads to inflammatory or anti-inflammatory cytokines and promotes chemotaxis of the stimulated cells $[5,7]$. MSCs, activated by TLR ligands, leading to modulation of differentiation, mobilization, migration, proliferation, recruitment, and immunosuppression capacities [7, 9]. The secretion pattern of lipopolysaccharide (LPS) treated cells appears to favor pro-inflammatory mediators, whereas polyinosinic-polycytidylic acid [poly(l:C)] mediated secretion patterns seem to favor antiinflammatory mediators [5]. LPS or poly $(1: C)$ are MSC agonists for TLR4 and TLR3, respectively. Invitro TLR3 stimulation has also been shown to stimulate the migration of MSCs [5, 7].
Granulocyte colony-stimulating factor (G-CSF) induces mobilization, proliferation, and differentiation of hematopoietic stem cells $[10,11]$. G-CSF-induced hematopoietic progenitor cell (HPC) mobilization from bone marrow has been attributed to changes in the bone marrow microenvironment, including activation of osteoclasts, upregulation of IL8, and metalloproteinases (MMPs) [11, 12]. G-CSF also affects T-cell and antigen-presenting cell function and suppresses the adaptive immune system [10]. The mechanisms of G-CSF induced immunomodulation in humans is not clearly defined. Immunomodulatory or immune suppressive effects of G-CSF may be attributed, at least partially, to its interaction with the MSCs. G-CSF has been shown to upregulate TLR2 expression on G-CSF mobilized peripheral blood stem cells (PBSC) and held responsible for rapid engraftment after allogeneic PBSC transplantation [13]. The results of the observational and clinical studies have suggested the contribution of G-CSF in the regenerative process after injury [14]. Also, G-CSF induced mobilization of MSCs (in addition to HPCs) from the bone marrow has been suggested $[15,16]$. It has been demonstrated that MSCs contribute to G-CSF-induced HPC mobilization from marrow by activating MMPs or by inducing partial depletion of B lymphocytes and osteal macrophages in the bone marrow $[17,18]$. However, it has remained unclear whether G-CSF may induce MSC mobilization from the marrow to aid in the repair process after injury.

Based on the previous studies showing G-CSF has a role in immunomodulation, engraftment, HPC migration, the present study was planned to investigate TLR2, TLR3, and TLR4 expression on the MSCs derived from G-CSF-exposed marrow donors $[13,19,20]$. Researchers evaluate TLR driven effects on MSCs. The results have shown that TLR 2 and 4 stimulation induces MSCs towards a proinflammatory phenotype (MSC-1), whereas TLR3 induces immunosuppression (MSC-2) [5]. Therefore, TLR2, TLR3, and TLR4 expressions of MSCs after agonist stimulation were also studied whether G-CSF was exposed or not. 


\section{MATERIALS and METHODS}

\section{Human Bone Marrow Samples}

The study was conducted at Ankara Children's Hematology and Oncology Research Hospital. Bone marrow samples were provided from the hematopoietic stem cell transplantation (HSCT) donors of our patients. G-CSF was administered to donors in whom the estimated mononuclear cell count in the harvested product would be lower than $<2 \times 10^{8} / \mathrm{kg}$ host weight and not suitable for PBSC collection. G-CSF was administered subcutaneously (lenograstim, Granocyte ${ }^{\circledR}$ ) at $10 \mu \mathrm{g} /$ $\mathrm{kg} /$ dose/daily, consecutively for three days. Bone marrow was collected on the fourth day. Three $\mathrm{mL}$ bone marrow samples were collected from bone marrow transplant donors during the stem cell harvest procedure. Bone marrow MSCs obtained from nine healthy bone marrow donors exposed to in-vivo G-CSF and G-CSF untreated controls $(n=9)$ were used for the studies. Median ages of in-vivo G-CSF-exposed or unexposed bone marrow donors were 9.2 years $(1.1-16.5), 9.5$ years $(3.3-17.2)$, respectively. Institutional Ethical and Review Board approval was obtained (11042012-1), and informed consent was signed by parents and/or donors.

\section{Mesenchymal Stem Cell Expansion and Characterization}

Mononuclear cells were isolated with the density gradient centrifugation method using ficoll (density: $1.077 \mathrm{~g} / \mathrm{mL}$ ) (Biochrom, Germany). Mononuclear cells were plated in $75 \mathrm{~cm}^{2}$ flasks at least $20 \times 10^{6}$ cell with $10 \mathrm{~mL}$ growth medium [DMEM-LG (Biochrom, Germany), 10\% fetal bovine serum (FBS) (Biochrom, Germany), 1\% antibiotic (Penicillin-Streptomycin, Biochrom, Germany)] and incubated in $37^{\circ} \mathrm{C}, 5 \% \mathrm{CO}_{2}$ (Galaxy $170 \mathrm{R}$, Eppendorf Company, Germany). MSCs were isolated and expanded in-vitro to achieve a sufficient number of cells for intended studies. MSCs were plated in 6 well plates for TLR expression and characterization studies. Early passage cells <Passage 3 were used to maintain TLR profiles of the MSCs as close as possible to in-vivo state. MSCs were visualized by microscopy to observe fibroblast-like morphology. In-vitro differentiation capacity of MSCs towards adipogenic and osteogenic lineages was tested. Briefly, Adipogenic Stimulatory Supplements (Human) (Stemcell Technologies, Canada) induction was used for adipogenic, and Osteogenic
Stimulatory Supplements (Human) (Stemcell Technologies, Canada) was used to induce osteogenic differentiation. Oil Red $\mathrm{O}$ and Alizarin Red stainings were used to verify respectively, adipogenic and osteogenic differentiation capacity of MSCs, at day 21 of induced cultures. MSCs were also tested for positive staining of HLA ABC, CD90, CD73, CD44, and CD49e (BD Biosciences, USA) and negative antibody staining for $C D 34, C D 3, C D 4$, and HLA DR (BD Biosciences, USA). Flow cytometry with BD-FACSAria (BD Biosciences, USA) was used for surface phenotyping of MSCs.

International Society for Cellular Therapy definition was used to prove these isolated and proliferated cells as MSCs [21]. In-vivo G-CSF exposed MSCs were readily expanded, and their spindle-shaped morphology was maintained. Also, the adipogenic and osteogenic differentiation capacities of G-CSF exposed cells were preserved and the surface markers indicating stromal phenotypes are shown.

\section{TLR Ligands and Priming Protocol}

MSCs were plated in 6 well-plates at least $0.75-1$ $\mathrm{x} 10^{5}$ cell / well with $2 \mathrm{~mL}$ growth medium (DMEMLG, $10 \% \mathrm{FBS}$ ) and incubated in $37^{\circ} \mathrm{C}, 5 \% \mathrm{CO}_{2}$. Following expansion for about one week, MSCs have grown to $60-70 \%$ confluency before initiating the TLR priming. Escherichia coli-pure LPS (InvivoGen, USA) and poly(I:C) (InvivoGen, USA) were used as agonists for TLR4, TLR2, and TLR3, respectively. TLR ligands were used in the following concentrations: $10 \mu \mathrm{g} / \mathrm{mL}$ (high) - $10 \mathrm{ng} / \mathrm{mL}$ (low) LPS, $25 \mu \mathrm{g} / \mathrm{mL}$ (high) $-1 \mu \mathrm{g} / \mathrm{mL}$ (low) poly(l:C) $[5,19]$. TLR agonists were added to the fresh growth medium, and the cells were exposed to the agonists for 4 hours; they were then washed. After that, they were incubated for 16 hours without the agonists, then analyzed. The same protocol was implemented for 24 hours. However, LPS or poly(l:C) agonist stimulation of MSCs for 24 hours diminished the MSCs counts and failed to analyze by flow cytometry.

\section{TLR Expression by Flow Cytometry}

TLR2, TLR3, TLR4 protein expression of the G-CSFexposed and unexposed bone marrow-derived MSCs measured by flow cytometry with humanspecific antibodies. A representative sample is presented in Figure 1. TLR agonists stimulated 

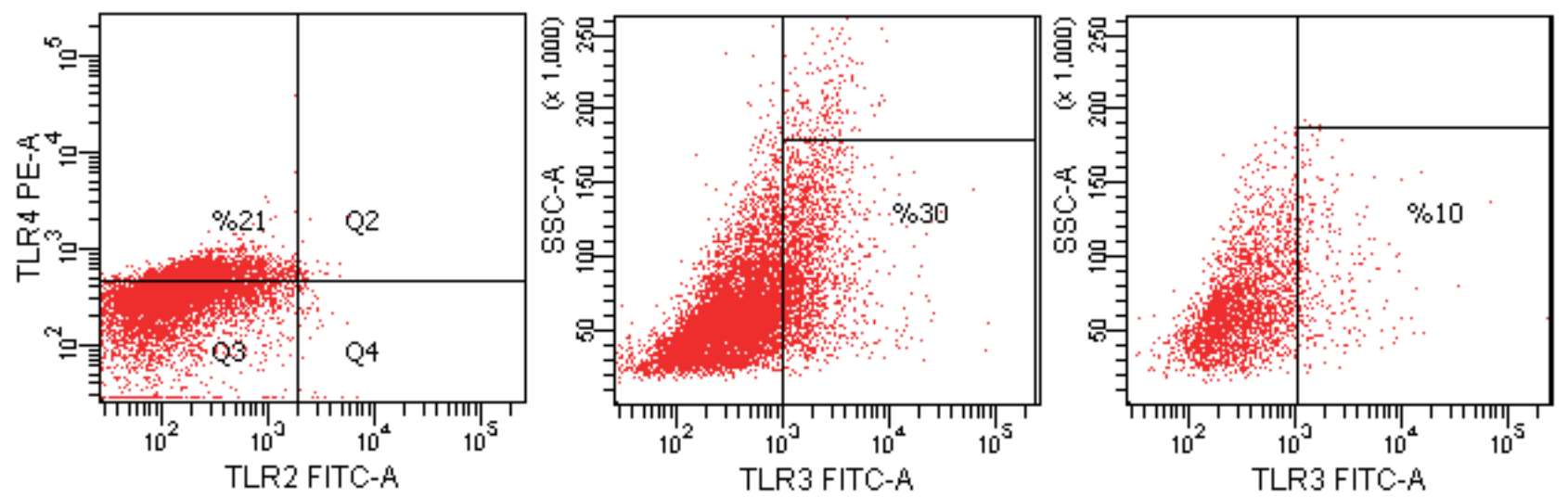

a) TLR2, TLR4 (First panel), and TLR3 expression (Middle panel), and TLR3 expression after low dose poly(l:C) treatment for 4 hours (Third panel) were represented respectively, which belongs to a G-CSF exposed donor.
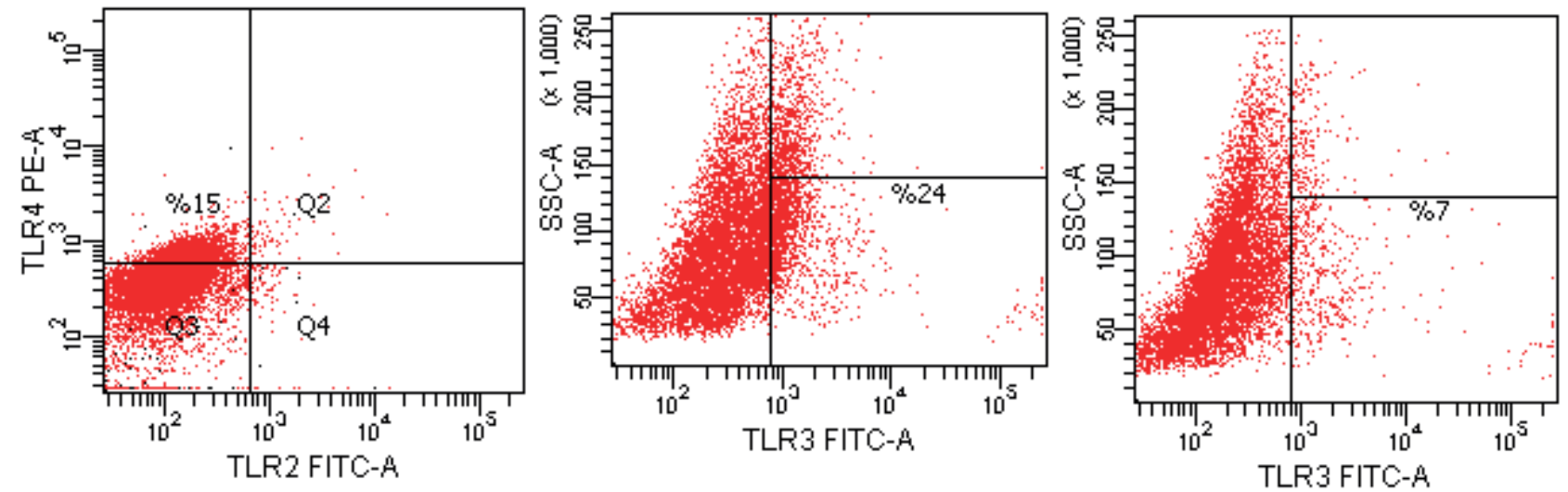

b) TLR2, TLR4 (First panel), and TLR3 expression (Middle panel), and TLR3 expression after low dose poly(l:C) treatment for 4 hours (Third panel) were represented respectively, which belongs to a G-CSF unexposed donor.

Figure 1. MSCs were stained for cell surface expression of TLR2, TLR4, and intracellular expression of TLR3 and analyzed by flow cytometry (BDFACSAria). Representative Picture of MSCs belongs to one donor

MSCs with different dosages and periods to study TLR ligand binding capacity and alteration of the TLR profiles.

MSCs were harvested and analyzed by flow cytometry with a BD-FACSAria (BD Biosciences, USA). BD-FACS Diva software was used for analysis (BD Biosciences, USA). Unstained cells were used to eliminate autofluorescence of these cells (isotype controls). Anti-Human Toll-like receptor 2 (CD282) FITC (eBioscience, USA) and anti-human Toll-like receptor 4 (CD284) PE (eBioscience, USA) were used for cell surface TLR2 and TLR4 receptor analysis. Purified monoclonal antibody to human TLR3 labeled with FITC (Invivogen, USA) was used for intracellular antibody staining. Fixation and permeabilization of the cells achieved with the Perm II solution (BD Biosciences, USA), instructed by the manufacturer. Flow cytometry analysis was stopped when 10000 events were counted. TLR2, TLR3, and TLR4 expression and mean fluorescence intensity (MFI) were calculated.

\section{Statistical Analysis}

Data were represented as the mean and standard error of the mean. A comparison between LPS and poly $(I: C)$ groups was analyzed by the Friedman T-test (SPSS Software v 15.0, IBM, USA). Comparison between in vivo G-CSF treated or untreated groups were analyzed by Mann-Whitney $U$ Test (SPSS Software $v 15.0$, IBM, USA). Values of $p<0.05$ were considered statistically significant.

\section{RESULTS}

MSCs, isolated from healthy human bone marrow, showed low TLR2 expression. Flow cytometric TLR2 expression on MSCs was $3.3 \% \pm 1.5$ (MFI value: $198.7 \pm 27.7)$ in G-CSF unexposed samples. Similarly, in vivo, G-CSF treated MSCs showed low TLR2 expression $(2.99 \% \pm 1.27$, MFI value: $248.2 \pm 33.2)$. In vivo, G-CSF treatment did not effect MSC's TLR2 expression and MFI ( $p>0.05)$. LPS was used as an 
agonist, but no significant difference was observed in the TLR2 expression and MFI, both in the G-CSF exposed and unexposed groups ( $p>0.05$ ) (Data not represented).

MSCs showed higher expression levels of TLR3, which was shown in Table 1. Flow cytometric TLR3 expression on MSCs was $32.6 \% \pm 10$ (MFI value: $1110 \pm 333$ ) in G-CSF unexposed samples. In vivo, G-CSF treated MSCs also expressed TLR3 $(28 \% \pm 11.2$, MFI value: $1058 \pm 386)$. No significant difference was observed in the TLR3 expression and MFI among the G-CSF exposed and unexposed groups ( $p>0.05$ ). Poly $(I: C)$ agonist was used for TLR3 stimulation. Regarding G-CSF unexposed MSCs group, TLR3 expression and MFI were decreased as $68 \%$ and $57 \%$, respectively, by applying a low dose agonist for 4 hours. In the G-CSF exposed MSCs group, TLR3 expression and MFI were decreased as
$63 \%$ and $19 \%$, respectively, by applying low dose poly $(\mathrm{l}: \mathrm{C})$ agonist for 4 hours. TLR3 expression and MFI values of the poly $(I: C)$ treated or untreated MSCs are presented in Table 1.

MSCs showed low TLR4 expression, which was shown in Table 1. Flow cytometric TLR4 expression on MSCs was $9 \% \pm 4.9$ (MFI value: $249 \pm 23.3$ ) in G-CSF unexposed samples. Similarly, in vivo, G-CSF treated MSCs showed low TLR4 expression $(4.3 \% \pm 2.3$, MFI value: $246 \pm 38.4)$. No significant difference was observed in the TLR4 expression and MFI among the G-CSF exposed and unexposed groups ( $p>0.05$ ). LPS was used as an agonist for TLR4. No significant difference was observed in the expression and MFI of TLR4, both in the G-CSF exposed and unexposed groups ( $p>0.05$ ). TLR4 expression and MFI values of the LPS treated or untreated MSCs are recorded in Table 1.

Table 1. TLR3 and TLR4 expression and MFI values of the G-CSF exposed and unexposed MSCs, and poly(I:C) - LPS simulation results

\begin{tabular}{|c|c|c|c|c|c|}
\hline \multirow{2}{*}{ Group } & \multicolumn{2}{|c|}{ G-CSF + } & \multicolumn{2}{|c|}{ G-CSF - } & \multirow{2}{*}{$p$} \\
\hline & $\mathrm{n}$ & $\overline{\mathrm{X}} \pm \mathrm{S}_{\overline{\mathrm{x}}}$ & $\mathrm{n}$ & $\bar{X} \pm S_{\bar{x}}$ & \\
\hline \multicolumn{6}{|c|}{ TLR3 Expression (\%) } \\
\hline w/o* agonist & 9 & $28.0 \pm 11.2$ & 9 & $32.6 \pm 10^{a}$ & $>0.05$ \\
\hline Poly 4 High d. ${ }^{+}$ & 9 & $17.8 \pm 10.7$ & 9 & $22.4 \pm 8.3^{b}$ & $>0.05$ \\
\hline Poly 4 Low d." & 8 & $10.3 \pm 4.9$ & 7 & $10.5 \pm 4.9^{b}$ & $>0.05$ \\
\hline $\mathrm{p}$ & & $>0.05$ & & $<0.05$ & \\
\hline \multicolumn{6}{|l|}{ TLR3 MFI } \\
\hline w/o* agonist & 9 & $1058 \pm 386^{a}$ & 9 & $1110 \pm 333^{a}$ & $>0.05$ \\
\hline Poly 4 High $\mathrm{d}^{+}{ }^{+}$ & 9 & $1061 \pm 422^{a}$ & 9 & $1232 \pm 419^{a}$ & $>0.05$ \\
\hline Poly 4 Low d. ${ }^{\ddagger}$ & 8 & $856 \pm 383^{b}$ & 7 & $472 \pm 128^{b}$ & $>0.05$ \\
\hline$p$ & & $<0.05$ & & $<0.05$ & \\
\hline \multicolumn{6}{|c|}{ TLR4 Expression (\%) } \\
\hline w/o* agonist & 9 & $4.3 \pm 2.3$ & 9 & $9 \pm 4.9$ & $>0.05$ \\
\hline LPS 4 High d..$^{\S}$ & 9 & $5.2 \pm 3$ & 9 & $16.3 \pm 7.4$ & $>0.05$ \\
\hline LPS 4 Low d.॥ & 8 & $3 \pm 1.4$ & 7 & $2.3 \pm 0.8$ & $>0.05$ \\
\hline $\mathrm{p}$ & & $>0.05$ & & $>0.05$ & \\
\hline \multicolumn{6}{|l|}{ TLR4 MFI } \\
\hline w/o* agonist & 9 & $246.6 \pm 38.4$ & 9 & $249.5 \pm 23.3$ & $>0.05$ \\
\hline LPS 4 High d. ${ }^{\S}$ & 9 & $297.5 \pm 61.2$ & 9 & $293.4 \pm 39.3$ & $>0.05$ \\
\hline LPS 4 Low d. $\|$ & 8 & $303.1 \pm 48.9$ & 7 & $280.1 \pm 48.5$ & $>0.05$ \\
\hline$p$ & & $>0.05$ & & $>0.05$ & \\
\hline
\end{tabular}

a,b: Difference is significant between varied letters in the same column

${ }^{*}$ w/o: Without

${ }^{+}$Poly 4 High d.: High dose poly $(\mathrm{l}: \mathrm{C})$ treatment for 4 hours

₹ Poly 4 Low d.: Low dose poly $(I: C)$ treatment for 4 hours

${ }^{5}$ LPS4 High d.: High dose LPS treatment for 4 hours

" LPS4 Low d.: Low dose LPS treatment for 4 hours 


\section{DISCUSSION}

Toll-like receptors are vital in coordinating MSC's responses to various stimuli $[7,9]$. It has been shown that TLR 2 and TLR4 stimulation induces MSCs towards a pro-inflammatory phenotype (MSC-1), whereas TLR3 induces immunosuppression (MSC2) [5]. TLR 3 has also been implicated in stem cell migration [5]. Here, we hypothesized that TLR profiles of MSCs might contribute to the stem/ progenitor cell mobilization potential and the immunomodulatory effects induced by G-CSF administration. Thus, we investigated Toll-like receptors' expression on mesenchymal stem cells due to in-vivo exposure to G-CSF with or without exposure of the cells to TLR agonists.

TLR levels of MSCs have been studied in several studies. Tomchuck et al. reported TLR2, 3, and 4 on MSCs [19]; Wang et al. showed TLR2, 4 on MSCs by flow cytometry [22]. Romieu-Mourez et al. showed MSCs express TLR3, 4 mRNA but lack TLR2. Also, they showed TLR3 expression on MSCs by flow cytometry, whereas TLR4 expression was low, as noted in the present study [23]. Besides, TLR expression was shown on MSCs by immunocytochemical analysis and by PCR analysis $[5,19,24,25]$. Additionally, the tissue origin of MSCs can affect their functions, mainly through TLRs [26]. A study compared MSCs from two different sources, including olfactory ecto-MSCs and adipose tissue MSCs. They showed both MSCs types express TLR3 and TLR4 in different expression levels by flow cytometry [26]. Human bone marrow-derived MSCs were reported to express TLR3 protein, but low expression of TLR2,4 by flow cytometry in the present study. Also, this was supported by a low amount of absolute MSCs carrying TLR2,4 by flow cytometry. In general, the results were inconsistent, particularly for TLR2 expression on MSCs. This may be attributed to the difference of the MSC source (human, mouse), the tissue of origin (bone marrow, adipose tissue), or the techniques used (PCR, flow cytometry, immunocytochemistry).

G-CSF exposed PBSC transplants have been shown to suppress the cellular immune response in recipients [20]. However, unstimulated T cells do not have a G-CSF receptor [20]. This finding suggests that G-CSF effects on T lymphocytes may be elicited through indirect mechanisms, perhaps through MSCs or other cells' involvement. In the present study, both G-CSF exposed and unexposed MSCs showed low expression of the pro-inflammatory TLR4 but higher levels of the immunosuppressive TLR3, suggesting that marrow-derived MSCs may be more prone to immunosuppressive rather than a pro-inflammatory state. In-vivo G-CSF administration to donors did not affect the expression of TLR2,4 on culture-expanded MSCs. Researchers suggest that MSCs may play a role in G-CSF induced HPC mobilization through different mechanisms involving MMP activation or depletion of $B$ cells and macrophages $[7,17,18]$. On the other hand, G-CSF induced MSCs mobilization from bone marrow is implicated in regeneration after injury [14]. Therefore, TLR3 expression on G-CSF exposed MSCs, and its agonist response may suggest that TLR3 may be a potential candidate molecule in MSC migration, and immunomodulation. However, in the present study, in-vivo exposure to G-CSF did not affect the TLR3 expression and MFI levels of MSCs. The studies on MSCs were performed on passage 3 MSCs, in which the effect of in-vivo GCSF has been lost; thus may not represent a real in-vivo situation. On the other hand, the number of MSCs on fresh bone marrow samples is too low to study TLR expression and be culture-expanded.Therefore, further studies upon ex-vivo G-CSF exposure and/ or molecular studies on sorted marrow samples may help. Joo et al. reported that G-CSF directly upregulates TLR2 expression on G-CSF mobilized PBSCs responsible for their rapid engraftment [13]. In the present study, TLR2 expression was shallow both in G-CSF-exposed and unexposed MSCs.

In contrast, MSCs expressed TLR3 as a potential molecule to be involved in mobilization. However, TLR3 agonist induction of MSCs was associated with a significant drop in TLR3 expression and MFI, which was more profound in G-CSF unexposed cells, suggesting an intrinsic potential for stimulation. But, it is considered that the culture expansion of MSCs might have abolished some properties and may not reflect the in-vivo state.

MSCs were stimulated by TLR agonists to study alteration of the TLR profiles. LPS was used as an agonist for TLR2 and TLR4. No significant difference was observed in the expression of TLR2 and TLR4, both in the G-CSF exposed and unexposed MSCs. Tomchuck et al. showed downregulation of the 
TLR3, 4 expressions stimulated by Poly $(\mathrm{l}: \mathrm{C})$ and LPS, respectively [19]. Downregulation of the TLR proteins was attributed to receptor activation, internalization, and degradation [19]. Our results were at least partially attributed to the low levels of TLR2 and TLR4 on marrow-derived MSCs. However, it should be kept in mind that MSCs may respond to different agonists other than LPS, which were not tested in the present study. Poly $(I: C)$ agonist induction of MSCs was associated with a significant drop in TLR3 expression and MFI, which was more profound in G-CSF unexposed cells, suggesting an intrinsic potential for stimulation. Our findings are consistent with the report of Tomchuck et al. [19]. High expression of TLR3 on MSC and inducibility with its agonist poly $(I: C)$ might indicate MSC's role in both immunosuppressive and migration effects. Studies also supported that TLR3 agonists stimulate MSCs signaling pathways more prominently than other TLRs $[5,19]$. TLR3, which is linked with cell migration, may potentially affect the migratory function of bone marrow MSCs, thus contributing to hematopoiesis and regeneration. It is well known that G-CSF mainly effects HPCs. A study suggests that G-CSF also augments the migration of MSCs from bone marrow [15]. G-CSF induced HPC mobilization involves osteoclast activation, IL-8 upregulation, MMP, and CXCR4SDF-1 inhibition [12]. The role of TLR3 in G-CSF induced migration of MSCs has not been studied. In the present study, TLR3 expression and MFI of in vivo G-CSF treated MSCs were decreased by $63 \%$ and $19 \%$, respectively, by applying poly $(\mathrm{l}: \mathrm{C})$ agonist for 4 hours. These results suggested that G-CSF exposure may not affect TLR expression and MFI of MSCs significantly. However, it may modulate the MSCs' response to agonists. The changes in TLR3 expression and MFI after TLR3 ligand stimulation was different between G-CSF treated or untreated MSCs. This finding may suggest a role for TLR3 in G-CSF-induced migration and mobilization from bone marrow. The study's limitations are those lack of variable methods to reveal TLR on MSCs or a few agonists' implementations to stimulate TLR might influence the results. Also, the lack of functional studies of TLR was a drawback of the study.

In conclusion, this study is novel to disclose the effect of in-vivo G-CSF exposure on healthy human bone marrow-derived MSCs and their TLR expressions. Delineation of the mechanisms in further investigations may reveal the immunomodulatory, migratory, and invasive properties of TLR agonist or G-CSF stimulated MSCs. TLR3 agonist applications to MSCs may enlighten the mechanism of G-CSF mediated cell migration and mobilization. Additionally, MSCs' therapeutic efficiency may be augmented by TLR3 modulations.

\section{CONFLICT Of INTEREST STATEMENT}

The authors have no conflict of interest to declare. 
[1] Horwitz EM, Le Blanc K, Dominici M, et al. International Society for Cellular Therapy. Clarification of the nomenclature for MSC: The International Society for Cellular Therapy position statement. Cytotherapy. 2005; 7: 393-5.

[2] Caplan Al. Mesenchymal stem cells. J Orthop Res. 1991;9:641-50.

[3] Aggarwal S, Pittenger MF. Human mesenchymal stem cells modulate allogeneic immune cell responses. Blood. 2005; 105: 1815-22.

[4] Yagi H, Soto-Gutierrez A, Parekkadan B, et al. Mesenchymal stem cells: Mechanisms of immunomodulation and homing. Cell Transplant. 2010; 19: 667-79.

[5] Waterman RS, Tomchuck SL, Henkle SL, et al. A new mesenchymal stem cell (MSC) paradigm: polarization into a pro-inflammatory MSC1 or an Immunosuppressive MSC2 phenotype. PLoS One. 2010; 5: e10088.

[6] Fu X, Liu G, Halim A, et al. Mesenchymal Stem Cell Migration and Tissue Repair. Cells. 2019; 8: 784.

[7] Shirjang S, Mansoori B, Solali S, et al. Toll-like receptors as a key regulator of mesenchymal stem cell function: An upto-date review. Cell Immunol. 2017; 315: 1-10.

[8] Fitzgerald KA, Kagan JC. Toll-like Receptors and the Control of Immunity. Cell. 2020; 180: 1044-1066.

[9] DelaRosa O, Lombardo E. Modulation of adult mesenchymal stem cells activity by toll-like receptors: implications on therapeutic potential. Mediators Inflamm. 2010; 2010: 865601.

[10] Anderlini P, Champlin RE. Biologic and molecular effects of granulocyte colony-stimulating factor in healthy individuals: recent findings and current challenges. Blood. 2008; 111: 1767-72.

[11] de Kruijf EFM, Fibbe WE, van Pel M. Cytokine-induced hematopoietic stem and progenitor cell mobilization: unraveling interactions between stem cells and their niche. Ann N Y Acad Sci. 2020; 1466: 24-38.

[12] Lapid K, Glait-Santar C, Gur-Cohen S, et al. Egress and Mobilization of Hematopoietic Stem and Progenitor Cells: A dynamic multi-facet process (December 10, 2012), StemBook, ed. The Stem Cell Research Community, StemBook, doi/10.3824/stembook.1.91.1, http://www. stembook.org.

[13] Joo YD, Lee WS, Won HJ, et al. Upregulation of TLR2 expression on G-CSF-mobilized peripheral blood stem cells is responsible for their rapid engraftment after allogeneic hematopoietic stem cell transplantation. Cytokine. 2011; 54: 36-42.

[14] Kastrup J, Ripa RS, Wang Y, et al. Myocardial regeneration induced by granulocyte-colony-stimulating factor mobilization of stem cells in patients with acute or chronic ischaemic heart disease: a non-invasive alternative for clinical stem cell therapy? Eur Heart J. 2006; 27: 2748-54.

[15] Yu Q, Chen L, You Y, et al. Erythropoietin combined with granulocyte colony stimulating factor enhances MMP-2 expression in mesenchymal stem cells and promotes cell migration. Mol Med Rep. 2011; 4: 31-6.

[16] Gilevich IV, Fedorenko TV, Pashkova IA, et al. Effects of Growth Factors on Mobilization of Mesenchymal Stem Cells. Bull Exp Biol Med. 2017; 162: 684-686.

[17] Ponte AL, Ribeiro-Fleury T, Chabot V, et al. Granulocytecolony-stimulating factor stimulation of bone marrow mesenchymal stromal cells promotes CD34+ cell migration via a matrix metalloproteinase-2-dependent mechanism. Stem Cells Dev. 2012; 21: 3162-72.

[18] de Kruijf EFM, van Hengel I, Perez-Galarza JM, et al. Mesenchymal Stromal Cells Enhance G-CSF-Induced Mobilization of Hematopoietic Stem- and Progenitor Cells. Blood. 2013; 122: 2460.

[19] Tomchuck SL, Zwezdaryk KJ, Coffelt SB, et al. Toll-like receptors on human mesenchymal stem cells drive their migration and immunomodulating responses. Stem Cells. 2008; 26: 99-107.

[20] Franzke A, PiaoW, Lauber J, et al. G-CSF as immune regulator in T cells expressing the G-CSF receptor: implications for transplantation and autoimmune diseases. Blood. 2003; 102: 734-9.

[21] Dominici M, Le Blanc K, Mueller I, et al. Minimal criteria for defining multipotent mesenchymal stromal cells. The International Society for Cellular Therapy position statement. Cytotherapy. 2006; 8: 315-7.

[22] Wang $X$, Cheng $Q$, Li L, et al. Toll-like receptors 2 and 4 mediate the capacity of mesenchymal stromal cells to support the proliferation and differentiation of $\mathrm{CD}_{3} 4^{+}$ cells. Exp Cell Res. 2012; 318: 196-206.

[23] Romieu-Mourez R, François M, Boivin MN, et al. Cytokine modulation of TLR expression and activation in mesenchymal stromal cells leads to a pro-inflammatory phenotype. J Immunol. 2009; 182: 7963-73.

[24] Pevsner-Fischer M, Morad V, Cohen-Sfady M, et al. Toll-like receptors and their ligands control mesenchymal stem cell functions. Blood. 2007; 109: 1422-32.

[25] Hwa Cho H, Bae YC, Jung JS. Role of toll-like receptors on human adipose-derived stromal cells. Stem Cells. 2006; 24: 2744-52.

[26] Jafari M, Asghari A, Delbandi AA, et al. Priming TLR3 and TLR4 in human adipose- and olfactory mucosa-derived mesenchymal stromal cells and comparison of their cytokine secretions. Cytotechnology. 2020; 72: 57-68. 